\title{
Facial and Simple Synthesis of Some New (Pyrazole and Triazole) Coumarin Derivatives
}

\author{
H.A. Latif, M.A. Barsy, E.A. Elrady \\ Department of Chemistry, Faculty of Science, Aswan University, Aswan, Egypt.
}

\begin{abstract}
H-coumarin-3-carbohydrazide (2) which prepared from the reaction of ethyl-2-oxo-2Hcoumarin-3-carboxylate (1) with hydrazine hydrate in ethanol containing a catalytic amount of piperidine mixture consider a good and available starting intermediate for synthesis of series of functionalized coumarins. So, compound (2) was utilized as a key for the synthesis of some new (pyrazole, triazole)-2H-coumarin-2-one derivatives by the reaction with some selected reagents.
\end{abstract}

Keywords: (pyrazole, triazole)-coumarin-2-one derivatives. The structures of these compounds were established by elemental analysis, IR and ${ }^{I} H$ NMR spectral analysis.

\section{Introduction}

The coumarin derivatives are prominent natural products, which are widely distributed among many plants [1]. They have considerable biological importance, especially as potentially useful pesticides [2, 3], inhibitors of cell proliferation and potential anticancer therapeutics [4]. The coumarin derivatives was found as new PET agents for imaging of apoptosis in cancer [5], potent inhibitory activities in enzymatic and cellular assays and good selectivity to MMP-2 and MMP-9 [6], dye compounds [7-9], potent antileishmanial activity [10]. Antirhinovirus activity in cell cultures, several compounds were also proved to be both potent and selective HRV1B inhibitors [11]. The coumarin compounds display high antifungal and antibacterial activities [12-15]. Also, coumarin derivatives was found as anti-picornavirus capsid-binders [16], and exhibited profound antioxidant activities [17]. Infinitively, the coumarin-3-carboxamide derivatives were found as potent inhibitors of AKR1B10 [18].

In addition, derivatives of coumarin are important natural products possessing a wide range of valuable physiological activities [19]. And also, they represent useful synthetic building blocks in organic and medicinal chemistry [20-25]. The coumarinol moiety of vitamin E (a-tocopherol) exhibits anti-androgen properties [26], coumarinyl derivatives also display significant cytotoxic activity against cancer cells [27].

The importance of the coumarin nucleus is evidenced by the continued appearance of new and improved methods for their synthesis, despite the several existing methods for the synthesis of coumarin derivatives [2842], there is still demand for general strategies, which can efficiently provide variously substituted coumarin systems.

\section{Matrials And Methods}

All melting points are measured using Galenkamp melting point apparatus and are uncorrected. Elemental analysis was carried out at the Microanalytical Center of Cairo University by an automatic analyzer (Heraeus). IR ( $\mathrm{KBr}$ pellets) spectra were determined in $1650 \mathrm{FT}-9 \mathrm{R}$ instrument (Cairo University). ${ }^{1} \mathrm{H}$ NMR spectra were accomplished using $300 \mathrm{MHz}$ NMR Spectrometer and mass spectroscopy were recorded on GCMS-QP-1000 EX spectrometer (Cairo University). 


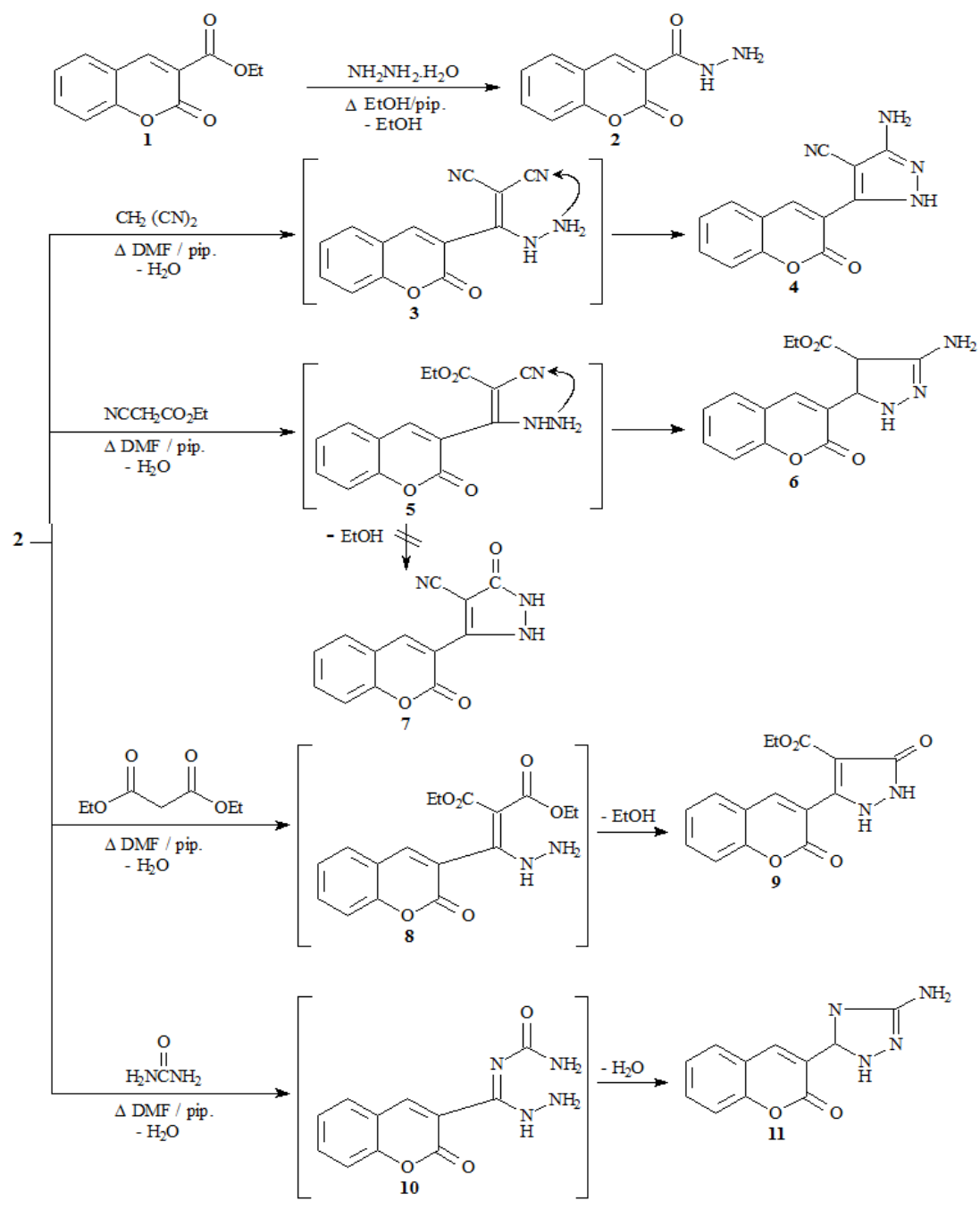

Scheme 1

\section{Synthesis of 2-oxo-2H-coumarin-3-carbohydrazide (2):}

A mixture of $1(0.44 \mathrm{~g}, 2 \mathrm{mmol})$ and hydrazine hydrate $(0.1 \mathrm{ml}, 2 \mathrm{mmol})$ in ethanol $(50 \mathrm{ml})$ containing piperidine $(0.1 \mathrm{ml})$ was refluxed for 5 hours, the solvent was evaporated under vaccum. The residue was triturated with methanol, the resulting product was collected by filtration, washed with methanol and crystallized from ethanol. The results are registered in Table $(1,2)$.

\section{Synthesis of 3-(1H-3-amino-4-cyano-pyrazole-5-yl)-2H-coumarin-2-one (4):}

A mixture of $2(0.41 \mathrm{~g}, 2 \mathrm{mmol})$ and malononitril $(0.13 \mathrm{~g}, 2 \mathrm{mmol})$ in $30 \mathrm{ml}$ DMF containing $(0.1 \mathrm{ml})$ piperidine was refluxed for 6 hours, the solvent was evaporated under vaccum, the residue was triturated with ice/water and the resulting product was collected by filtration, washed with $100 \mathrm{ml}$ cold water and crystallized from dioxane. The results are registered in Table $(1,2)$.

Synthesis of 3-(1H-3-amino-4-ethyl carboxy-pyrazole-5-yl)-2H-coumarin-2-one (6):

A mixture of $2(0.41 \mathrm{~g}, 2 \mathrm{mmol})$ and ethylcyanoacetate $(0.23 \mathrm{ml}, 2 \mathrm{mmol})$ in $30 \mathrm{ml}$ DMF containing $(0.1 \mathrm{ml})$ piperidine was refluxed for 5 hours. The solvent was evaporated under vacuum, the residue was triturated with methanol, the resulting product was collected by filtration, washed with methanol and crystallized from dioxane. The results are registered in Table $(1,2)$.

\section{Synthesis of 3-(1,2,3H-4-ethyl carboxy-3-oxo-pyrazole-5-yl)-2H-coumarin-2-one (9):}

A mixture of $2(0.41 \mathrm{~g}, 2 \mathrm{mmol})$ and diethylmalonate $(0.32 \mathrm{ml}, 2 \mathrm{mmol})$ in $30 \mathrm{ml}$ DMF containing $(0.1$ $\mathrm{ml}$ ) piperidine was refluxed for 5 hours. The solvent was evaporated under vacuum, the residue was triturated with methanol, the resulting product was collected by filtration, washed with methanol and crystallized from dioxane. The results are registered in Table $(1,2)$. 
Synthesis of 3-(1H-3-amino-1, 2, 4-triazole-5-yl)-2H-coumarin-2-one (11):

A mixture of $2(0.41 \mathrm{~g}, 2 \mathrm{mmol})$ and urea $(0.10 \mathrm{ml}, 2 \mathrm{mmol})$ in $30 \mathrm{ml}$ DMF containing $(0.1 \mathrm{ml})$ piperidine was refluxed for 5 hours. The solvent was evaporated under vacuum, the residue was triturated with methanol, the resulting product was collected by filtration, washed with methanol and crystallized from dioxane. The results are registered in Table $(1,2)$.

Table 1: Characterization of the prepared compounds

\begin{tabular}{|c|c|c|c|c|c|c|c|c|c|c|}
\hline \multirow{3}{*}{$\begin{array}{l}\text { Comp. } \\
\text { No }\end{array}$} & \multicolumn{3}{|c|}{ Nature of products } & \multirow{3}{*}{$\begin{array}{l}\text { Molecular } \\
\text { formula (M.Wt) }\end{array}$} & \multicolumn{6}{|c|}{ Analysis \% } \\
\hline & \multirow[t]{2}{*}{ Colour } & \multirow[t]{2}{*}{ Yield \% } & \multirow{2}{*}{$\begin{array}{l}\text { M.p. } \\
{ }^{\circ} \mathrm{C}\end{array}$} & & \multicolumn{3}{|c|}{ Calculated } & \multicolumn{3}{|c|}{ Found } \\
\hline & & & & & $\mathrm{C}$ & $\mathrm{H}$ & $\mathrm{N}$ & $\mathrm{C}$ & $\mathrm{H}$ & $\mathrm{N}$ \\
\hline 2 & yellow & 65 & $\begin{array}{l}136- \\
138\end{array}$ & $\begin{array}{l}\mathrm{C}_{10} \mathrm{H}_{8} \mathrm{~N}_{2} \mathrm{O}_{3} \\
(204.05)\end{array}$ & 58.86 & 3.95 & 13.73 & 58.92 & 3.98 & 13.85 \\
\hline 4 & $\begin{array}{l}\text { Plate } \\
\text { Brown }\end{array}$ & 62 & $\begin{array}{l}220 \\
222\end{array}$ & $\begin{array}{l}\mathrm{C}_{13} \mathrm{H}_{8} \mathrm{~N}_{4} \mathrm{O}_{2} \\
(252.23)\end{array}$ & 61.90 & 3.20 & 22.21 & 61.97 & 3.25 & 22.25 \\
\hline 6 & yellow & 65 & $\begin{array}{l}260 \\
263\end{array}$ & $\begin{array}{l}\mathrm{C}_{15} \mathrm{H}_{13} \mathrm{~N}_{3} \mathrm{O}_{4} \\
(299.28)\end{array}$ & 60.20 & 4.38 & 14.04 & 60.26 & 4.42 & 14.11 \\
\hline 9 & yellow & 63 & $\begin{array}{l}230 \\
233\end{array}$ & $\begin{array}{l}\mathrm{C}_{15} \mathrm{H}_{12} \mathrm{~N}_{12} \mathrm{O}_{5} \\
(300.27)\end{array}$ & 60.20 & 4.03 & 9.33 & 60.27 & 4.10 & 9.38 \\
\hline 11 & yellow & 62 & $\begin{array}{l}186 \\
188\end{array}$ & $\begin{array}{l}\mathrm{C}_{11} \mathrm{H}_{8} \mathrm{~N}_{4} \mathrm{O}_{2} \\
(288.21)\end{array}$ & 57.89 & 3.53 & 24.55 & 57.95 & 3.62 & 24.62 \\
\hline
\end{tabular}

Table 2: IR and ${ }^{1} \mathrm{H}$ NMR (Mass) Spectral Data of the Prepared Compounds.

\begin{tabular}{|c|c|c|}
\hline Comp. No & IR Spectrum $\left(\mathrm{KBr}, \mathrm{Cm}^{-1}\right)$ & ${ }^{1}$ H NMR Spectrum (DMSO, $\left.\delta\right) ; \&$ (Mass data) \\
\hline 2 & $\begin{array}{l}1682 \text { (amidic CO), } 1730 \text { (lactone } \mathrm{C}=\mathrm{O} \text { ), } \\
3073(\mathrm{NH}), 3321\left(\mathrm{NH}_{2}\right)\end{array}$ & $\begin{array}{l}\delta 2\left(\mathrm{~d}, 2 \mathrm{H}, \mathrm{NH}_{2}\right), 7.02-7.27(\mathrm{~m}, 5 \mathrm{H}, \mathrm{Ar}-\mathrm{H}), 8(\mathrm{t}, 1 \mathrm{H}, \mathrm{NH}), 8.37(\mathrm{~s}, \mathrm{H}, \\
\mathrm{CH}) . \\
\mathrm{M}^{+}: 204\end{array}$ \\
\hline 6 & $1698(\mathrm{C}=\mathrm{O}), 2208(\mathrm{C} \equiv \mathrm{N}), 3402(\mathrm{NH})$ & $\begin{array}{l}1.3\left(\mathrm{t}, 3 \mathrm{H}, \mathrm{CH}_{3}\right), 4\left(\mathrm{~s}, 2 \mathrm{H}, \mathrm{NH}_{2}\right), 4.29\left(\mathrm{q}, 2 \mathrm{H}, \mathrm{CH}_{2}\right), 7.02-7.72(\mathrm{~m}, 5 \mathrm{H} \text {, } \\
\mathrm{Ar}-\mathrm{H}) . \\
\mathrm{M}^{+}: 299\end{array}$ \\
\hline 9 & $1269(\mathrm{C}-\mathrm{O})$ ester, $1698(\mathrm{C}=\mathrm{O}), 3430(\mathrm{NH})$ & $\begin{array}{l}1.30\left(\mathrm{t}, 3 \mathrm{H}, \mathrm{CH}_{3}\right), 2(\mathrm{~d}, 1 \mathrm{H}, \mathrm{NH}), 4.19\left(\mathrm{q}, 2 \mathrm{H}, \mathrm{CH}_{3}\right), 7.02-7.38(\mathrm{~m}, 5 \mathrm{H}, \\
\mathrm{Ar}-\mathrm{H}), 8(\mathrm{~d}, 1 \mathrm{H}, \mathrm{NH}) . \\
\left(\mathrm{M}^{+2}\right): 302\end{array}$ \\
\hline 11 & $3125(\mathrm{NH}), 3425\left(\mathrm{NH}_{2}\right)$ & $\begin{array}{l}4.01\left(\mathrm{~s}, 2 \mathrm{H}, \mathrm{NH}_{2}\right), 7.02-7.72(\mathrm{~m}, 5 \mathrm{H}, \mathrm{Ar}-\mathrm{H}), 13.5(\mathrm{~s}, 1 \mathrm{H}, \mathrm{NH}) \\
\mathrm{M}^{+}: 228\end{array}$ \\
\hline
\end{tabular}

\section{Results And Disscution}

2-Oxo-2H-coumarin-3-carbohydrazid (2) was prepared from the reaction of ethyl-2-oxo-2H-coumarin3-carboxylate (1) with hydrazine hydrate in ethanol containing amount of piperdine as a catalyst [1].

Compound (2) was reacted with malononitril, ethylcyanoacetate, diethylmalonate and urea to gave new compounds $(4,6,9,11)$ via intermediates $(3,5,8,10)$ respectively.

The cyclization of intermediates $(3,5)$ proceeded by nucleophilic addition of amino group into cyano group to afford compounds $(4,6)$. Compound (9) were formed by elimination of ethanol molecule from intermediate (8). Compound (11) afforded due to condensation reaction of intermediate (10). The structures of the synthesized compounds were established based on analytical and spectral analysis.

\section{Conclusion}

2-Oxo-2H-coumarin-3-carbohydrazide was synthesis and reacted with some selected reagent to give some new (pyrazole and triazole)-2H-coumarin-2-one derivative, structures of these compounds were established by elemental analysis, IR and ${ }^{1} \mathrm{H}$ NMR spectral analysis.

[1]. Ellis, G. P. 1977, Coumarins, Chromanones, and Chromones. The Chemistry of Heterocyclic Compounds; Wiley: New York, Vol. 31.

[2]. Bowers, W. S. 1985, In Comprehensive Insect Physiology, Biochem Pharmacology; Gilbert, L. I., Kerkut, G. A., Eds.; Pergamon: Oxford, Vol. 8, p 551

[3]. Bowers, W. S.; Ohta, T.; Cleere, J. S.; Marsella, P. A., 1976, Science, 193, 542.

[4]. William, K.; Shailaja, K.; Songchun, J.; Hong, Z.; Jianghong, Z.; Shaojuan, J.; Lifen, X.; Candace, C.; Re 'al, D.; Nancy, B.; Louis, V.; Sylvie, C.; Jennifer, D.; Giorgio, A.; Denis, L.; Serge, L.; Henriette, G.; Ben, T.; John, D.; Sui, X. C., 2005, Bioorg. Med. Chem. Lett., $15,4745$.

[5]. Mingzhang, G.; Min, W.; Kathy, D. M.; Gary, D. H.; QiHuang, Z., 2010, Applied Radiation and Isotopes, 68, 110.

[6]. Kwangwoo, C.; Song-Kyu, P.; Hwan, M. K.; Yongseok, C.; Myung-Hwa, K.; Chun-Ho, P.; Bo-Young, J.; Tae, G. C.; Hyun-Moo, C.; Hee-Yoon, L.; Sung, H. H.; Myung, S. K.; Ky-Youb, N.; Gyoonhee, H., 2008, Bioorg. Med. Chem. 16, 530.

[7]. $\quad$ Nicolaou, K. C.; Pfefferkorn, J. A.; Cao, G.-Q., 2000, Angew Chem. Int. Ed., 39, 734.

[8]. Nicolaou, K. C.; Cao, G.-Q.; Pfefferkorn, J. A., 2000, Angew Chem. Int. Ed., 39, 739.

DOI: $10.9790 / 5736-1005012326 \quad$ www.iosrjournals.org

25 |Page 
[9]. Maggiani, A.; Tubul, A.; Brun, P., 2000, Helv. Chim. Acta., 83, 650.

[10]. Tadigoppula, N.; Tanvir, K.; Shweta, N.; Neena, G.; Suman, G., 2005, Bioorg. Med. Chem., 13, 6543.

[11]. Cinzia, C.; Nicoletta, D., 2009, Bioorg. Med. Chem., 17, 3720.

[12]. Ahmed, M. M.; El-Saghier, M. B.; Naili, B. K.; Rammash, N. A.; Saleh, Khaled, M. K., 2007, Arkivoc, 83

[13]. $\quad$ Laurin, P.; Klich, M.; Dupis-Hamelin, C.; Mauvais, P.; Lassaigne, P.; Bonnefoy, A.; Musicki, B., 1999, Bioorg Med. Chem. Lett., 9, 2079.

[14]. Tarik, E. A.; Salah, A. A. A.; Hafez, M. E.; Faten, I. H.; Ali, Z. E., 2008, Turk J. Chem., 32, 365.

[15]. $\quad$ Tilak, R.; Richa, K. B.; Rakesh, K. S.; Vivek, G.; Deepak, S.; Mohan, P. S. I., 2009, Euro J. Med. Chem., 44, 3209.

[16]. Cinzia, C.; Nicoletta, D., 2010, Bioorg. Med. Chem., 18, 6480.

[17]. $\quad$ Okram, M. S.; Nepram, S. D.; Dhanaraj, S. T.; Gurumayum, J. S., 2010, Euro J. Med. Chem., 45, 2250.

[18]. Satoshi, E.; Toshiyuki, M.; Kazuo, K.; Hai-Tao, Z.; El-Kabbani, O.; Yukio, K.; Akira, H., 2010, Bottom of Form Bioorg. Med. Chem., 18, 2485.

[19]. Horton, D. A.; Bourne, G. T.; Smythe, M. L., 2003, Chem. Rev., 103, 893.

[20]. Nohara, A.; Kuriki, H.; Saijo, T.; Sugihara, H.; Kanno, M.; Sanno, Y., 1977, J. Med. Chem., 20, 141

[21]. Nohara, A.; Umetani, T.; Ukawa, K.; Sanno, Y., 1974, Chem. Pharm. Bull., 22, 2959.

[22]. Ukawa, K.; Ishiguro, T.; Wada, Y.; Nohara, A., 1986, Heterocycles, 24, 1931.

[23]. Hsung, R. P., 1997, J. Org. Chem., 62, 7904.

[24]. Hsung, R. P.; Zificsak, C. A.; Wei, L.-L.; Zehnder, L. R.; Park, F.; Kim, M.; Tran, T. T. 1999, J. Org. Chem. $64,8736$.

[25]. Sosnovskikh V. Ya.; Irgashev, R. A.; Levchenko, A. A., 2008, Tetrahedron, 64, 6607.

[26]. Van, H. N.; Bettina, A.; Peter, L., 2006, Tetrahedron 62, 7674.

[27]. Tilak, R.; Richa, K. B.; Ashish, K.; Madhunika, S.; Saxena, A. K.; Ishar, M. P. S., 2010, Euro J. Med. Chem., 45, 790.

[28]. Dorta, R. L.; Martin, A.; Sua '́rez, E.; Betancor, C., 1997, J Org. Chem., 622273.

[29]. Vyacheslav, Ya.; Vladimir, S. M.; Roman, A. I., 2006, Tetrahedron Lett., 47, 8543.

[30]. Brian, D.; Liren, Z.; Junko, T.; Joseph, P.; Sarah, H.; Brett, C.; Christopher, E. H.; Jenny, W.; Christi, N.; Ajay, M.; David, S.; Warren, W.; Val, S. G., 2006, Bioorg. Med. Chem. Lett., 16, 4237.

[31]. Ying, W. G.; Yong, L. S.; Hong, B. L.; Min, S., 2006, Tetrahedron, 62, 5875.

[32]. Seiji, Y.; Mikiko, M.; Yohei, M.; Masahiro, M.; Yoshiro, H., 2004, Tetrahedron Lett., 45, 6971.

[33]. Galal, E. H. E.; Ahmed, H. H. E., 1990, Bull. Chem. Soc. Jpn., 63, 1230.

[34]. Rafat, M. M.; Hoda, Z. S.; Mohamed, H. E., 1992, Gazz. Chim. Italy, 122, 41.

[35]. Sergiy, M. K.; Igor, E. B.; Konstantyn, M. S.; Valentyn, P. C.; Yaroslav, V. B., 2000, Molecules, 5, 1146.

[36]. (a) Bacon, E. R.; Daum, S. J.; Singh, B., 1996, PCT Int Appl Wo 96 28, 429; (b) Bacon, E. R.; Daum, S. J.; Singh, B., 1996, Chem. Abstr., 125, 23, 301016b.

[37]. Saied, A. E.; Galal, H. S.; Ahmed, F., 2004, Acta Pharm., 54, 143.

[38]. Jose ', M. Q.; Carlos, P.; Liliana, G.; Rau '1, I.; Anabel, P.; Francisca, A '.; Manuel, L.; Sanmartı 'n, R. R., 2003, Euro J. Med. Chem., 38, 265.

[39]. Ahmed, M. M. E., 2002, Molecules, 7, 756.

[40]. Vishnu J. R.; Vidyohama D. A. J. V., 1987, J Heterocyclic Chem., 24, 1435.

[41]. Abu-Elmaati, T. M.; El-Taweel, F. M.; Elmougi, S. M.; Elagamey, A., 2004, J. Heterocyclic Chem. 41, 655.

[42]. Mostafa, M. K.; Ashraf, H. F.; Abd El-Wahab, F. A.; Eid, A. M. E. 2002, IL Farmaco, 57, 715.

[43]. Atul, M.; Alpeshkumar, M.; Jitender, V.; Vijay, V.; Arum, M.; Kuldip, U.; Evans, C.; Anamik, S., 2008, Eur J Med Chem., 43, 2395-2403. 